\title{
Interaction can hurt - Exploring gesture-based interaction for users with Chronic Pain
}

\author{
G. Michael Poor \\ Computer Science, Baylor University \\ Waco, TX, USA \\ michael_poor@baylor.edu
}

\author{
Alvin Jude \\ Ericsson Research, Ericsson, Inc. \\ Silicon Valley, USA \\ alvinjude@acm.org
}

\begin{abstract}
Chronic Pain is a universal disorder affecting millions of people, influencing even the most basic decisions in their lives. With the computer becoming such an integral part of our society and the ever-expanding interaction paradigm, the need to explore potential computer interactions for people with Chronic Pain has only increased. In this paper we explore the used of gesture-based interaction as a medium with which these users can perform the base operations of computer interaction. We show that, for gestural pointing and selection, modeling users' interaction space and multimodel interaction performed the best in terms of throughput.
\end{abstract}

\section{CCS CONCEPTS}

- Human-centered computing $\rightarrow$ Empirical studies in accessibility.

\section{KEYWORDS}

Gesture Interaction; Chronic Pain; Accessibility

\section{ACM Reference Format:}

G. Michael Poor and Alvin Jude. 2019. Interaction can hurt - Exploring gesture-based interaction for users with Chronic Pain. In Symposium on Spatial User Interaction (SUI '19), October 19-20, 2019, New Orleans, LA, USA. ACM, New York, NY, USA, 5 pages. https://doi.org/10.1145/3357251.3357589

\section{INTRODUCTION}

Pain warns us that something isn't quite right, a normal sensation triggered in the nervous system to alert someone to possible injury and the need to take care of one's self. When pain persists, it robs people of productivity, well-being, and, for many with extended illness, their very lives. When pain signals keep firing in the nervous system for weeks, months, even years, this is called "Chronic Pain" [31] (pain lasting longer than 3 months). Currently, Chronic Pain has become a universal disorder, a serious and costly public health issue, with a range of prevalence in the general public from 7\% [9] to 55\% [2]. Regardless of where the actual number falls, Chronic Pain is a huge challenge, causing a reduced ability to work and difficulties in performing everyday activities [21]. Additionally, the issue of Chronic Pain will continue to grow as $80 \%$ to $85 \%$ of

Permission to make digital or hard copies of all or part of this work for personal or classroom use is granted without fee provided that copies are not made or distributed for profit or commercial advantage and that copies bear this notice and the full citation on the first page. Copyrights for components of this work owned by others than the author(s) must be honored. Abstracting with credit is permitted. To copy otherwise, or republish, to post on servers or to redistribute to lists, requires prior specific permission and/or a fee. Request permissions from permissions@acm.org.

SUI '19, October 19-20, 2019, New Orleans, LA, USA

() 2019 Copyright held by the owner/author(s). Publication rights licensed to ACM. ACM ISBN 978-1-4503-6975-6/19/10_..\$15.00

https://doi.org/10.1145/3357251.3357589 persons experience a significant health problem that predisposes them to Chronic Pain after age 65. This pain tends to be less head, abdominal, and chest pain and more joint pain [15], which affects even the most basic of daily activities.

One area of computer interaction that relies heavily on a user's ability to move and interact is touchless gestural interaction. Touchless gestural interaction has become increasingly popular with the introduction of commodity hardware such as the Xbox Kinect and the Leap Motion. Gestural recognition has traditionally been the focus of gesture research, while implementations have focused on coarse gestural recognition (i.e. shape identification). However, commodity gestural recognition hardware has become increasingly more precise with devices demonstrated to be accurate up to a submillimeter level [20] with low latency [10]. Due to this increased precision, studies have started looking at gestures not simply for coarse actions, but for more fine use cases such as cursor navigation [10,25], a prime tenet in standard graphical interaction.

The standard method of interaction for gestural input involves the user holding their arms out in mid-air. This results in fatigue [36, 40] which is commonly referred to as "Gorilla Arm Syndrome" [11]. This gestural fatigue problem has been investigated and solutions developed for the general population. For example a gestural interaction approach called "Personal Space" [25] allowed the user to rest their elbow during interaction as well as define their area of interaction. This rested gestural interaction attempted to reduce repetitive wrist movement compared to the mouse, which has been strongly associated with hand impairments [41], by shifting primary motions from the wrist and hand to larger muscle groups such as the forearm and shoulder areas. Several studies were conducted on standard users using this approach and uncovered a few benefits: fatigue was greatly reduced [25], performance degradation when switching hands was minor when compared to the touchpad and mouse [24], and pairing gestural cursor navigation with speech based selection yielded no significant loss in throughput [19]. Each result by itself is interesting, but the combination shows promise for users with Chronic Pain. Unfortunately, the techniques and workarounds that have been incorporated in standard gesture interaction, have never been applied or tested on a user population that feels fatigued, stress, and pain from movements that a "typical" user would not with the same amount of interaction.

In this paper we explore whether touchless gestural interaction is suitable for users with Chronic Pain. We divided the interaction into two problem spaces: 1.) Gestural Pointing - use of a user's body to control interaction location and direction and 2.) Gestural Selection - gestures used to hold, release, or select items within an interaction system. 


\section{RELATED WORK}

\subsection{Gestural Interaction}

Gestural Interaction is a technique that uses gestures from the body to interact with a computer. This type of interaction technique has been studied for over three decades since Richard Bolt's first implementation in "Put-That-There" [8]. Recent devices such as the Xbox Kinect, Leap Motion, and Myo Armband have gained popularity among researchers, with some capable of sub-millimeter accuracy in static situations [20]. These devices have demonstrated the potential use of gestural interfaces in medical professions that require sterile environments $[7,29,40]$, as an accessibility device for those with impairments $[4,19]$, and in mixed reality environments with head mounted displays [13]. [32] looked into the use of the Xbox Kinect for point-select tasks. The experiment utilized a 2D and 3D multi-directional task to evaluate the pointing device. The study found Kinect's throughput was lower for the 2D task, but outperformed the mouse in the 3D task. They also found that Fitts' law extended into gestures similarly to the mouse in the 2D task and at a higher rate in the 3D task.

The "Come As You Are" design principle [37] states that users should not be required to wear a glove or specific markers to interact with the system [40]. In gestural interaction this means a "barehanded interaction style" where users interact with the computer without any device or wires attached. This approach has been described to be superior to previously used gestural input devices where gloves are used [39]. This approach was also utilized to demonstrate human-computer interaction using gestures that are natural and relaxed [14].

2.1.1 Gestural Fatigue. Fatigue is one of the biggest issues with gestures after prolonged interaction [34]. A simple method of overcoming this issue is to allow users to rest their elbows on a chair armrest $[14,34]$. Brown et al. implemented this method to perform cursor navigation with 2 possible modes of input: the whole hand and finger pointing. Jude et al. implemented a very similar approach to the previous whereby cursor navigation was done with the whole hand, but the user's input space was first modeled during a calibration stage [25]. Guinness et al. [18] modeled the input space with a sphere as opposed to the previously used quadilateral [25]. All these approaches make the same claim: performing gestural interaction from a rested position results in a more comfortable interaction and reduces the fatigue inherent to gestural interaction.

2.1.2 Gestural Pointing. Recently touchless gestural pointing has been implemented in one of two general pointing methods. The first, "ray pointing," is a popular approach used by many designers [5, $10,23,28]$ which uses ray casting to determine where the user is pointing [23]. This method is rapid but inaccurate [12]. Conversely, 'whole hand pointing' directly maps the 2D movements of the hand (by dropping one dimension) to the 2D cursor on screen [10]. The user can then move their hand or finger within this navigation space to move the cursor on screen. This method has been shown $[10,12$, $25]$ to be both rapid and accurate, even without visual feedback [12]

2.1.3 Gestural Selection. We use the term "gestural selection" to mean hand gestures used to select or 'choose' virtual objects in $2 \mathrm{D}$ or $3 \mathrm{D}$. The two most popular and common forms of gestural selection are "grab" and "pinch" both of which have been used extensively [14, 17, 38, 42]. A third option is "grasp," [6, 27]. Brown found that the finger tap gesture, which was considered the closest gesture to a mouse selection, performed inadequately for use in their experiment [10]. To combat this, [10] implemented a bimanual selection method, where users used their other hand to press the space bar when the cursor was over the target. Other researchers $[22,25,33]$ used a 'hover-select' or 'dwell' method which required the user to hover over the target for between 250 and 1500 milliseconds.

\subsection{Chronic Pain Related Diseases and Conditions (CPD)}

Acute and chronic are the two basic types of pain and they differ greatly. While acute pain is a normal sensation triggered in the nervous system to alert an individual to possible injury and the need to take care of themselves, Chronic Pain is persistent. With Chronic Pain signals can keep firing in the nervous system for weeks, months, or even years [31]. People with Chronic Pain disorders are often saddled with impairment of physical and social functions, as well as sleep disturbance, anxiety and depressive disorders. These associated symptoms may have further negative impact on a patient's total life situation and might affect the patient as much as the pain [3]. There are dozens of potential Chronic Pain conditions, however, the conditions that would be most effected by the continued use of gesture interaction would be Osteoarthritis (OA), Rheumatoid arthritis (RA), Fibromyalgia syndrome (FMS), and Carpal Tunnel Syndrome (CTS). The prolonged and specific gesture use of hand, arm, shoulder, and upper back cause the aforementioned conditions the most stress. This is not an exhaustive Chronic Pain list, but does focus on conditions that would typically feel discomfort and pain in most of the major muscle and joints area that are attributed to gesture-based interaction. The amount of pain is correlated to how much users use the mouse; higher amounts of pain leads to lower usage [1].

\section{METHODS}

\subsection{Participants}

17 participants between the ages of 18 - 53 were recruited $(F=9$, $\mathrm{M}=8$ ). Participants' computer use ranged from 1 to 50 hours per week with a median of 30 hours. All participants were volunteers with previously diagnosed conditions.

\subsection{Apparatus}

A symmetrical mouse (Logitech M-U0032-O) allowed participants to use either hand, and the Leap Motion captured gestures. Participants were encouraged to position peripherals for their best comfort. Researchers adjusted the angle and position of the Leap Motion to maximize recognition and comfort.

\subsection{Gesture Pointing}

To evaluate Gestural Pointing we explored three conditions, a mouse (default) and two gestural (Rested-Uncalibrated [10], and Rested-Calibrated [25]). For Rested-Uncalibrated gestural interaction, participants were allowed to rest their elbow on a surface, 

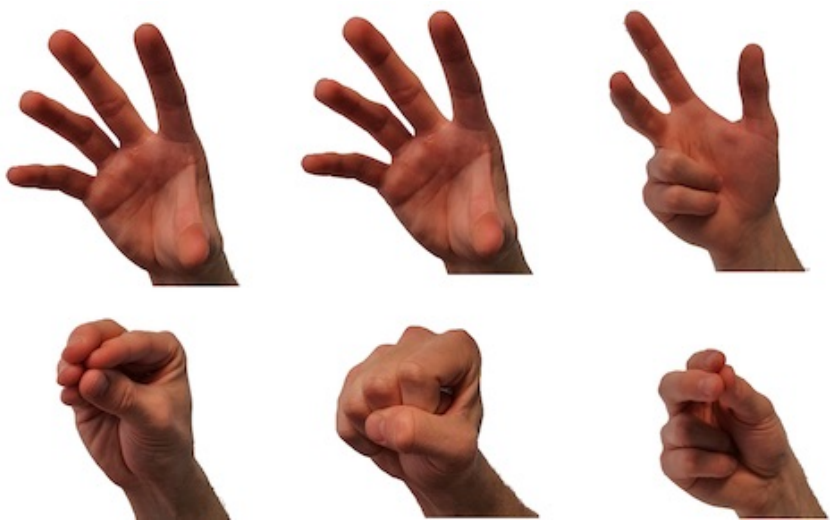

Figure 1: The gestures used for selection, with the open pose at the top and the closed pose at the bottom. From L-R: grasp, grab, pinch.

which is known to reduce fatigue. The cursor was linearly mapped to the interaction space and bounded to the length of the participants arm to ensure they would not have to lift their arm from the table to reach far ends of the screen. The Rested-Calibrated interaction is based on 'Personal Space' [25], the user definition of the four edges of interaction are used to build a quadilateral which are mapped to screen coordinates. The use of these two different methods allows us to explore if there is a benefit to modeling the user's interaction space, or if a simple linear-map would suffice.

\subsection{Gesture Selection}

To evaluate the Gestural Selection problem space, we investigated a proposed standard vocabulary for touchless gestural interaction for selection tasks - analogous to a drag-and-drop action performed with a mouse. Natural and intuitive gestures, as outlined in the literature review, were developed as well as a keyboard button input condition. The three versions of gesture interactions are called grab, pinch, and grasp (Figure 1).

3.4.1 Interaction Design. The design for selection gestures are based on the 4 usability principles for gestures proposed in [30]: 1) easy to perform and remember, 2) intuitive, 3) metaphorically and iconically logical towards functionality, and 4) ergonomic. [30] recommended a static pointing-like gesture for selection; however a later study indicated that a dynamic gesture is preferred over static gestures for selection [16]. Further, the grasp gesture was based on the R-family of gestures, which is used in real life for precision grip [26].

\subsection{Task}

For the gestural pointing task, standard ISO9241-9 Fitts evaluation with the ring-of-circles task was used with throughput which is a standard pointing performance metric [35]. Our task had three unique target distances (A): 350, 700, 1400 and three unique widths (W): 64, 96, 128, both measured in pixels. This produced index of difficulties between 1.90 and 4.52 bits, which falls within the lower end of the recommended 2-8 bits [35].
An ISO9241-9 evaluation task was also used during the gestural selection evaluation. However, in this case only one target size (700 px) and width (96 px) was used for all interactions. Each subject was asked to perform the task using one of the gestural selection input methods: grab, pinch, grasp, and multimodal (button).

\subsection{Procedure}

The participants were first shown a 1-minute video detailing the pointing task. Once completed the participants were shown both a short video explaining the the mouse interaction and a longer, 3 minute video outlining the gestural interaction. The gesture interaction video included an explanation of the calibration process. A researcher was present to address participants questions following the video.

When the gestural pointing task began, participants would perform the task once using each method, totalling 3 tasks per round. Each task is done with a different task profile, ensuring participants would not be able to learn the task or guess the position of each target. A practice session consisting of 12 trials was performed before the full task of 70 trials. The 3 tasks in the first round were followed by a 2 minute break, after which participants were asked to perform the exact same tasks as before, in the same order, and on the same task profile. The training task was not present in the second round.

For the gestural selection task, participants would perform a similar task as the gestural pointing but with half the number of trials. After each task completion, the user would perform the task again using a different input method: grab, pinch, grasp, and a multimodal approach where a button was used to initiate selection with the other hand. The order of the input methods was randomized as to, once again, limit the chance that some form of learning effect would be present. To remain consistent, the "Rested \& Calibrated Interaction" from the previous portion was used for cursor control. Participants were encouraged to pause and rest for as long as they required between rounds. During these breaks, the experimenter would engage with the participants and collect their thoughts and impressions about the previous interaction.

Upon completion, participants were interviewed about their experience with the various interactions detailing their prior use of each device and their preferences, comfort, and fatigue experienced with those devices during the experiment. The entire experiment lasted between 45 to 60 minutes barring any extended break requests by the participants.

\section{RESULTS}

\subsection{Gestural Pointing}

Levene's test showed the variance for throughput was not equal $\mathrm{F}(2,48)=5.9, \mathrm{p}=.005$. Hence Welch ANOVA was used for hypothesis testing, which showed there was a statistical significance on the effect of interaction on throughput, $\mathrm{F}(2,30)=5.90, \mathrm{p}<.001$. A GamesHowell posthoc test showed there was a statistical difference between Mouse and Gestural: Uncalibrated $(\mathrm{p}<.001)$, and Mouse and Gestural: PersonalSpace $(\mathrm{p}<.001)$.

Figure 2 is a Fitts regression which visualizes the impact of Index of Difficulty on Movement Time. Both gestural interactions had Y-intercepts closer to zero: 200 for uncalibrated and 236 for 

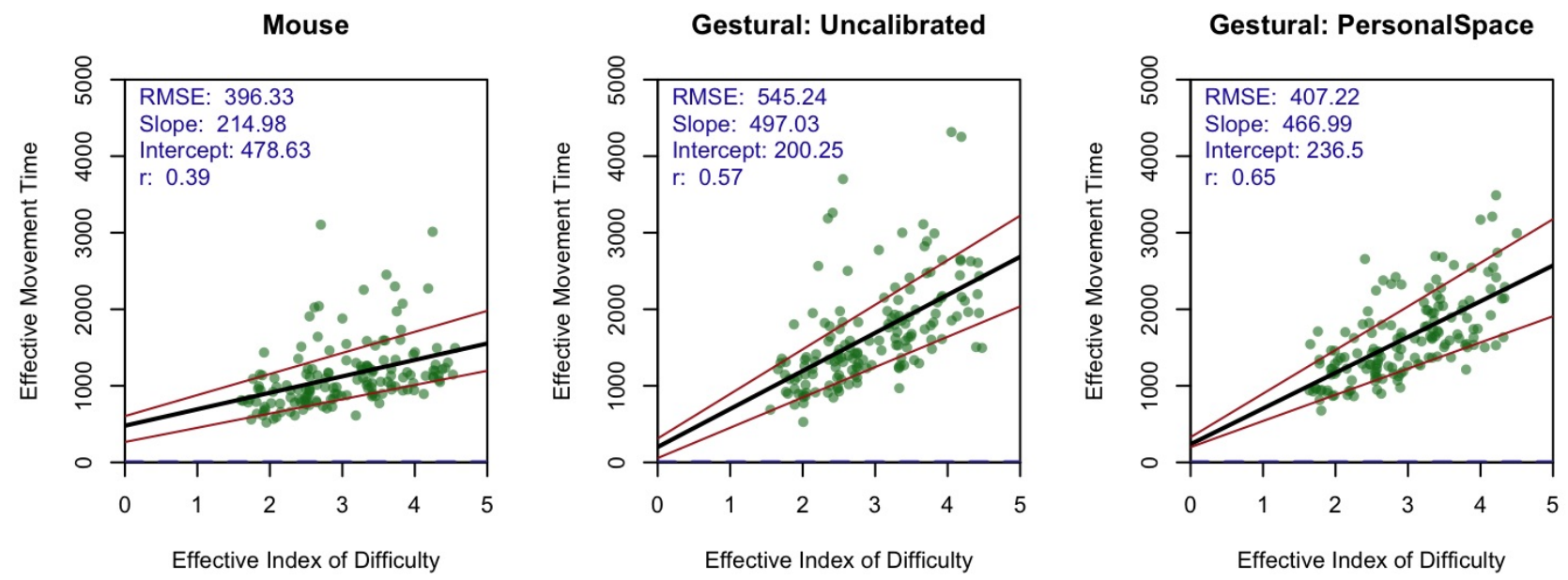

Figure 2: Visualization of the Fitts task per interaction based on. The black line is regression over the means while the red lines are quantile regression lines at .159 and .841 , hence $66 \%$ of the points fall within the red lines.

PersonalSpace, the mouse was at 479. Gestural: PersonalSpace had higher goodness of fit measured in Pearson's $r$ (.65) followed by Gestural: Uncalibrated (.57) and the mouse (.39). The model errors calculated in Root Mean Squared Errors (RMSE) was lowest on mouse (396) followed by Gestural: PersonalSpace (407) and Gestural: Uncalibrated (545).

\subsection{Gestural Selection}

A one-way repeated measures ANOVA showed that there was a statistical significant difference on the effect of interaction on throughput, $\mathrm{F}(3,48)=7.44, \mathrm{p}<.001$. Posthoc tests with Tukey's HSD showed a statistical difference between multimodal and the other three interactions (all $\mathrm{p}<.001$ ) but no significant differences between any of the others (grab, grasp, and pinch). This significant difference can be contributed to a few factors that occurred during the gesture selection task. The first of which was the accuracy of the gesture actions at each of the targets. For non-Chronic Pain users, these actions pose little to no problem in terms of repetitive action. However, for these participants the combination of fatigue and simplification of actions due to pain aversion caused their individual actions to miss the targets, requiring more time and movement to complete each task. Each participant, regardless of number of breaks requested, noted that these factors contributed to their performance degradation during their exit interviews.

4.2.1 Qualitative Results. On both the exit surveys and interviews, participants were overwhelmingly in favor of the multimodal input method. All participants noted that multimodal input provided the best perceived accuracy as well as an experience that was more familiar. In addition, when using the gestural interaction methods, participants had increasing difficulty holding their hands steady when performing the individual input gestures causing the cursor to move during target acquisition and the target to be initially missed. Another consistent issue with the gesture input methods was that in some cases the interaction caused participants' Chronic Pain to "flair up" due to small repetitive motions. This was especially evident in participants where wrist and hand pain was common (carpal tunnel and arthritis).

\section{DISCUSSION AND CONCLUSION}

The mouse had the best throughput while both gestural interactions had no significant difference. However, gestural interactions had a better Fitts' model in terms of RMSE and intercept. We believe this indicates gestural interaction could be better generalized to the population of participants with pain. Between the two gestural methods, PersonalSpace had better RMSE, Slope, and Pearson's r, which we believe demonstrates a benefit to modelling the individuals' interaction space, inline with previous research. Both gestural interactions also had higher slopes indicating smaller and/or further targets become harder to reach. This could perhaps be addressed by modifying the GUI to have targets that are larger for our target group.

The qualitative results also indicate that our users want the ability to re-position their hands constantly as this helps with pain conditions. This forced recalibration during gestural pointing, which reduced the overall experience. Future research should consider how the users interaction space can be re-modelled automatically without the need for manual calibration.

In conclusion, this research explored how gestural interaction can be suitable for people with Chronic Pain conditions and found that gestural pointing benefits from modeling users' interaction space. Additionally, a multimodal approach where the non-gesturing hand presses a button is better for selection. Our research indicates that these approaches could improve gestural interaction for people with Chronic Pain issues. While these findings are informative, future investigation is needed to investigate ways of combining these approaches without the need for a dual arm approach.

\section{ACKNOWLEDGMENTS}

This material is based upon work supported by the National Science Foundation under Grant No. 1551531. 


\section{REFERENCES}

[1] Johan Hviid Andersen, Mette Harhoff, Søren Grimstrup, Imogen Vilstrup, Christina Funch Lassen, Lars PA Brandt, Ann I Kryger, Erik Overgaard, Kasper D Hansen, and Sigurd Mikkelsen. 2008. Computer mouse use predicts acute pain but not prolonged or chronic pain in the neck and shoulder. Occupational and environmental medicine 65, 2 (2008), 126-131.

[2] H Ingemar Andersson, Göran Ejlertsson, Ido Leden, Claes Rosenberg, and others. 1993. Chronic pain in a geographically defined general population: studies of differences in age, gender, social class, and pain localization. The Clinical journal of pain 9, 3 (1993), 174-182.

[3] Paulin Andréll, Tomas Schultz, Kaisa Mannerkorpi, Phdlena Nordeman, Mats Börjesson, and Clas Mannheimer. 2014. Health-Related Quality of Life in Fibromyalgia and Refractory Angina Pectoris: A Comparison Between Two Chronic Non-Malignant Pain Disorders. Journal of Rehabilitation Medicine 46, 4 (2014), 341-347.

[4] Gilles Bailly, Jörg Müller, Michael Rohs, Daniel Wigdor, and Sven Kratz. 2012 ShoeSense: A New Perspective on Gestural Interaction and Wearable Applications. In Proceedings of the SIGCHI Conference on Human Factors in Computing Systems (CHI '12). ACM, New York, NY, USA, 1239-1248. DOI : http://dx.doi.org/10.1145/ 2207676.2208576

[5] Amartya Banerjee, Jesse Burstyn, Audrey Girouard, and Roel Vertegaal. 2011 Pointable: an in-air pointing technique to manipulate out-of-reach targets on tabletops. In Proceedings of the ACM International Conference on Interactive Tabletops and Surfaces. ACM, 11-20.

[6] Mark Becker, Efthimia Kefalea, Eric Maël, Christoph Von Der Malsburg, Mike Pagel, Jochen Triesch, Jan C. Vorbrüggen, Rolf P. Würtz, and Stefan Zadel. 1999. GripSee: A Gesture-Controlled Robot for Object Perception and Manipulation. Auton. Robots 6, 2 (April 1999), 203-221. DOI : http://dx.doi.org/10.1023/A 1008839628783

[7] Ali Bigdelou, Loren Schwarz, and Nassir Navab. 2012. An Adaptive Solution for Intra-operative Gesture-based Human-machine Interaction. In Proceedings of the 2012 ACM International Conference on Intelligent User Interfaces (IUI '12). ACM, New York, NY, USA, 75-84. DOI : http://dx.doi.org/10.1145/2166966.2166981

[8] Richard A Bolt. 1980. "Put-that-there": Voice and gesture at the graphics interface Vol. 14. ACM.

[9] D Bowsher, M Rigge, and L Sopp. 1991. Prevalence of chronic pain in the British population: a telephone survey of 1037 households. Pain Clinic 4, 4 (1991) 223-230.

[10] Michelle A. Brown, Wolfgang Stuerzlinger, and E. J. Mendonça Filho. 2014. The Performance of Un-instrumented In-air Pointing. In Proceedings of the 2014 Graphics Interface Conference (GI '14). Canadian Information Processing Society, Toronto, Ont., Canada, Canada, 59-66. http://dl.acm.org/citation.cfm?id=2619648. 2619659

[11] Tim Carmody. 2010. Why 'Gorilla Arm Syndrome' Rules Out Multitouch Notebook Displays. Wired, Oct 10 (2010).

[12] Andy Cockburn, Philip Quinn, Carl Gutwin, Gonzalo Ramos, and Julian Looser. 2011. Air pointing: Design and evaluation of spatial target acquisition with and without visual feedback. International fournal of Human-Computer Studies 69, 6 (2011), 401-414.

[13] Barrett Ens, Juan David Hincapié-Ramos, and Pourang Irani. 2014. Ethereal Planes: A Design Framework for 2D Information Space in 3D Mixed Reality Environments. In Proceedings of the 2nd ACM Symposium on Spatial User Interaction (SUI '14). ACM, New York, NY, USA, 2-12. DOI : http://dx.doi.org/10.1145/2659766. 2659769

[14] Dustin Freeman, Ramadevi Vennelakanti, and Sriganesh Madhvanath. 2012. Freehand pose-based Gestural Interaction: Studies and implications for interface design. In Intelligent Human Computer Interaction (IHCI), 2012 4th International Conference on. IEEE, 1-6.

[15] RM Gallagher, S Verma, and J Mossey. 2000. Chronic pain. Sources of late-life pain and risk factors for disability. Geriatrics 55, 9 (2000), 40-4.

[16] Sukeshini A. Grandhi, Gina Joue, and Irene Mittelberg. 2011. Understanding Naturalness and Intuitiveness in Gesture Production: Insights for Touchless Gestural Interfaces. In Proceedings of the SIGCHI Conference on Human Factors in Computing Systems (CHI '11). ACM, New York, NY, USA, 821-824. DOI : http: //dx.doi.org/10.1145/1978942.1979061

[17] Tovi Grossman, Daniel Wigdor, and Ravin Balakrishnan. 2004. Multi-finger Gestural Interaction with 3D Volumetric Displays. In Proceedings of the 17th Annual ACM Symposium on User Interface Software and Technology (UIST '04). ACM, New York, NY, USA, 61-70. DOI : http://dx.doi.org/10.1145/1029632.1029644

[18] Darren Guinness, Alvin Jude, G Michael Poor, and Ashley Dover. 2015. Models for rested touchless gestural interaction. In Proceedings of the 3rd ACM Symposium on Spatial User Interaction. ACM, 34-43.

[19] Darren Guinness, G Michael Poor, and Alvin Jude. 2014. Gestures with speech for hand-impaired persons. In Proceedings of the 16th international ACM SIGACCESS conference on Computers \& accessibility. ACM, 259-260.

[20] Jože Guna, Grega Jakus, Matevž Pogačnik, Sašo Tomažič, and Jaka Sodnik. 2014. An Analysis of the Precision and Reliability of the Leap Motion Sensor and Its
Suitability for Static and Dynamic Tracking. Sensors 14, 2 (2014), 3702-3720.

[21] Oye Gureje, Michael Von Korff, Gregory E Simon, and Richard Gater. 1998. Persistent pain and well-being: a World Health Organization study in primary care. Jama 280, 2 (1998), 147-151.

[22] Juan David Hincapié-Ramos, Xiang Guo, Paymahn Moghadasian, and Pourang Irani. 2014. Consumed Endurance: A Metric to Quantify Arm Fatigue of Midair Interactions. In Proceedings of the SIGCHI Conference on Human Factors in Computing Systems (CHI '14). ACM, New York, NY, USA, 1063-1072. DOI : http: //dx.doi.org/10.1145/2556288.2557130

[23] Ricardo Jota, Miguel A. Nacenta, Joaquim A. Jorge, Sheelagh Carpendale, and Saul Greenberg. 2010. A Comparison of Ray Pointing Techniques for Very Large Displays. In Proceedings of Graphics Interface 2010 (GI '10). Canadian Information Processing Society, Toronto, Ont., Canada, Canada, 269-276. http://dl.acm.org/ citation.cfm?id=1839214.1839261

[24] Alvin Jude, G Michael Poor, and Darren Guinness. 2014a. An evaluation of touchless hand gestural interaction for pointing tasks with preferred and nonpreferred hands. In Proceedings of the 8th Nordic Conference on Human-Computer Interaction: Fun, Fast, Foundational. ACM, 668-676.

[25] Alvin Jude, G. Michael Poor, and Darren Guinness. 2014b. Personal Space: User Defined Gesture Space for GUI Interaction. In CHI '14 Extended Abstracts on Human Factors in Computing Systems (CHI EA '14). ACM, New York, NY, USA, 1615-1620. DOI : http://dx.doi.org/10.1145/2559206.2581242

[26] Adam Kendon. 2004. Gesture: Visible action as utterance. Cambridge University Press.

[27] J-S Kim, K-H Park, J-B Kim, J-H Do, K-J Song, and Z Bien. 2000. Study on intelligent autonomous navigation of Avatar using hand gesture recognition. In Systems, Man, and Cybernetics, 2000 IEEE International Conference on, Vol. 2. IEEE, 846-851.

[28] I Scott MacKenzie and Shaidah Jusoh. 2001. An evaluation of two input devices for remote pointing. In Engineering for human-computer interaction. Springer, 235-250.

[29] Helena M Mentis, Kenton O'Hara, Abigail Sellen, and Rikin Trivedi. 2012. Interaction proxemics and image use in neurosurgery. In Proceedings of the SIGCHI Conference on Human Factors in Computing Systems. ACM, 927-936.

[30] Michael Nielsen, Moritz Störring, Thomas B Moeslund, and Erik Granum. 2004. A procedure for developing intuitive and ergonomic gesture interfaces for HCI. In Gesture-Based Communication in Human-Computer Interaction. Springer, 409420 .

[31] National Institute of Neurological Disorders, National Institutes of Health, and others. 2009. Pain: Hope through research. Journal of pain \& palliative care pharmacotherapy 23, 3 (2009), 307-322.

[32] Alexandros Pino, Evangelos Tzemis, Nikolaos Ioannou, and Georgios Kouroupetroglou. 2013. Using kinect for 2D and 3D pointing tasks: performance evaluation. In Human-Computer Interaction. Interaction Modalities and Techniques. Springer, 358-367.

[33] Lawrence Sambrooks and Brett Wilkinson. 2013. Comparison of gestural, touch, and mouse interaction with Fitts' law. In Proceedings of the 25th Australian Computer-Human Interaction Conference: Augmentation, Application, Innovation, Collaboration. ACM, 119-122.

[34] Jakub Segen and Senthil Kumar. 2000. Look Ma, No Mouse! Commun. ACM 43, 7 (July 2000), 102-109. DOI : http://dx.doi.org/10.1145/341852.341869

[35] R William Soukoreff and I Scott MacKenzie. 2004. Towards a standard for pointing device evaluation, perspectives on 27 years of Fitts' law research in HCI. International fournal of Human-Computer Studies 61, 6 (2004), 751-789.

[36] Vítor Teixeira. 2011. Improving elderly access to audiovisual and social media, using a multimodal human-computer interface. Ph.D. Dissertation. Faculdade de Engenharia, Universidade do Porto.

[37] Jochen Triesch and Christoph Von Der Malsburg. 1998. Robotic gesture recognition by cue combination. In Informatik' 98 . Springer, 223-232.

[38] Daniel Vogel and Ravin Balakrishnan. 2005. Distant Freehand Pointing and Clicking on Very Large, High Resolution Displays. In Proceedings of the 18th Annual ACM Symposium on User Interface Software and Technology (UIST '05). ACM, New York, NY, USA, 33-42. DOI : http://dx.doi.org/10.1145/1095034.1095041

[39] Christian Von Hardenberg and François Bérard. 2001. Bare-hand humancomputer interaction. In Proceedings of the 2001 workshop on Perceptive user interfaces. ACM, 1-8.

[40] Juan Pablo Wachs, Mathias Kölsch, Helman Stern, and Yael Edan. 2011. Visionbased hand-gesture applications. Commun. ACM 54, 2 (2011), 60-71.

[41] Gunilla Wieslander, D Norbäck, CJ Göthe, and L Juhlin. 1989. Carpal tunnel syndrome (CTS) and exposure to vibration, repetitive wrist movements, and heavy manual work: a case-referent study. British fournal of Industrial Medicine 46, 1 (1989), 43-47.

[42] Jamie Zigelbaum, Alan Browning, Daniel Leithinger, Olivier Bau, and Hiroshi Ishii. 2010. G-stalt: A Chirocentric, Spatiotemporal, and Telekinetic Gestural Interface. In Proceedings of the Fourth International Conference on Tangible, Embedded, and Embodied Interaction (TEI '10). ACM, New York, NY, USA, 261-264. DOI : http://dx.doi.org/10.1145/1709886.1709939 